\title{
ON THE NATURE OF TIME
}

\section{MURASKIN}

Physics Department University of North Dakota

Grand Forks, ND 58201

(Received August 26, 1988 and in revised form September 14, 1988)

ABSRACT. We study how the notion of time can affect the motion of particles within the No Integrability Aesthetic Field Theory. We show that the Minkowski hypothesis of treating $x^{4}$ as pure imaginary as well as the fourth component of vectors as pure imaginary, does not lead to different solutions provided we alter the sign of $\mathrm{dx}^{4}$ and certain origin point field components. We next show that it is possible to introduce time in the situation where all fields are real so that: (1) The field equations treat all coordinates the same way; (2) The "flow" concept is associated with time but not with space; (3) Data is prescribed at a single point rather than on a hypersurface as in hyperbolic theories. We study the lattice solution in the approximation that ignores zig-zag paths. This enables us to investigate the effect of a non-trivial superposition principle in the simplest way. We find that such a system, combined with our new approach to time, gives rise to an apparent infinite particle system in which particles can be looked at as not having well defined trajectories. This result is similar to what we obtained when we treated time in the same manner as the space variables in our previous work.

KEYWORDS AND PHRASES. Classical Field Theories 1980 AMS CLASSIFICATION CODE. $70 G 50$

\section{INTRODUCTION.}

The Aesthetic Field Theory treats all Cartegian tensors and the way these tensors change in a uniform way (Muraskin [1], Muraskin [2]). We obtain lattice solutions to the field equations in four dimensions in Muraskin [3]. In this situation the integrability equations are not satisfied. The lattice solutions are obtained by specifying an integration path. To get to any desired point we first integrate in $t$, then $z$, then $y$, and then $x$. We studied the motion of lattice particles when an integration path is specified as well as in the simplest approximation to the summation over path approach to the no integrability equations (Muraskin [4], Muraskin (5]). 
We shall define a field theory that leads to particles not having well defined trajectories as a $Q$ theory. This effect is to be intrinsic -- that 1s, we shall suppose that we are not dealing with a simllar situation as in statistical mechanics.

The hypothesis we make is that the way we integrate a no integrability theory can transform a C theory Into Q theory. A C theory 18 a fleld theory where particles have well defined trajectories. This hypothesis was found to be valid in the case of the "nafve" approximation of Muraskin [4]. We will find this to be the case here as well.

The basic problem addressed in this paper is the consequences of two different ways to view the concept of time such that time is not treated in the same manner as space in all respects. We will be investigating the notion of time within the context of the no integrability aesthetic fleld formilation.

The notion of time, as well as the notion of quantum theory, appear when we talk of free fields. For this reason we shall concern ourselves with lattice solutions. The lattice solution in the no integrability theory is akin to the free field situation in conventional fleld theorles, as it based on sine and cosine solutions along any path segment.

\section{MINKOWSKI VERSION OF AESTHETIC FIELD THEORY}

The aesthetic fleld equations are

$$
\frac{\partial \Gamma_{j k}^{1}}{\partial x^{1}}=\Gamma_{m k}^{1} \Gamma_{j 1}^{m}+\Gamma_{j m}^{1}-\Gamma_{j k}^{m} \Gamma_{m 1}^{1} .
$$

The index 1 runs from one to four and the fourth component is treated in the same manner as the first three coordinates.

In Minkowsi space, time is treated differently than space in the following way. The time coordinate is pure 1maginary. Vectors have their fourth component pure imaginary. We shall adopt this hypothesis in Aesthetic Field Theory. Following this procedure $\Gamma_{j k}^{1}$ is imaginary when the fourth Index appears an odd number of times. Otherwise $\Gamma_{j k}^{1}$ is pure real.

We have studied the following data in Muraskin [4],

$$
\begin{aligned}
& \mathrm{r}_{32}^{1}=1.0 \quad \mathrm{r}_{34}^{1}=-1.0 \\
& \mathrm{r}_{41}^{2}=1.0 \quad \mathrm{r}_{43}^{2}-1.0
\end{aligned}
$$

with the other $\Gamma_{\beta Y}^{\alpha}$ zero. This set of data leads, on using the same $e_{1}^{\alpha}$ as Muraskin [6], to a lattice in real Aesthetic Field Theory. So far as the numerical work tells us the lattice particles undergo ( $1 \mathrm{n}$ unison) simple harmonic motion in the $z$ direction.

We Introduce a complex $r_{\text {or }}^{\alpha}$ as follows:

with $A_{B Y}^{\alpha}, B_{B Y}^{\alpha}$ real.

$$
\Gamma_{B Y}^{\beta}=A_{B Y}^{\alpha}+1 B_{B Y}^{\alpha}
$$


Consider now the data

$$
\begin{array}{ll}
A_{32}^{1}=1.0 & B_{41}^{2}=-1.0 \\
B_{34}^{1}=-1.0 & B_{43}^{2}=1.0
\end{array}
$$

with the other $A_{B Y}^{\alpha}, B_{B Y}^{\alpha}$ zero.

We get a more complicated set of origin point data by using

$$
\Gamma_{j k}^{1}=e_{\alpha}^{1} e_{j}^{\beta} e^{\alpha}{ }_{k} \Gamma_{B \gamma}^{\alpha} \text {. }
$$

We write

$$
e_{1}^{\alpha}=h_{1}^{\alpha}+1 g_{1}^{\alpha}
$$

with $h^{\alpha}, g_{i}^{\alpha}$ rea 1 .

We have chosen

$$
h_{1}^{\alpha}=\left(\begin{array}{cccc}
.88 & -.42 & -.32 & 0 \\
.5 & .9 & -.425 & 0 \\
.2 & -.55 & .89 & 0 \\
0 & 0 & 0 & 1.01
\end{array}\right)
$$

and

$$
\mathrm{g}_{1}^{\alpha}=\left(\begin{array}{cccc}
0 & 0 & 0 & .22 \\
0 & 0 & 0 & .3 \\
0 & 0 & 0 & .6 \\
-.44 & -.16 & -.39 & 0
\end{array}\right)
$$

Such an $e^{\alpha}{ }_{1}$ will maintain the same structure for $A_{j k}^{1}$ as $A_{B \gamma}^{\alpha}$ and the same structure for $B_{j k}^{1}$ as $B_{B Y}^{\alpha}$ so far as reality properties are concerned. These reality properties are maintained by the field equations.

The set (2.2) gives a lattice. We have chosen the same $e_{1}^{\alpha}$ as in Muraskin [6]. The integration path is specified in the manner described in section 1.

We find that the set $(2.2-2.8)$ also gives rise to this same lattice solution.

In general, we find, that the Minkowski hypothesis does not lead to a different situation provided that we reverse the sign in

$$
\begin{gathered}
d x^{4}, B_{11}^{4}, B_{12}^{4}, B_{13}^{4}, B_{21}^{4}, B_{22}^{4}, B_{23}^{4}, B_{31}^{4}, B_{32}^{4}, \\
B_{33}^{4}, A_{44}^{1}, A_{44}^{2}, A_{44}^{3}, g_{1}^{4}, g_{2}^{4}, g_{3}^{4}
\end{gathered}
$$

while the other $\Gamma_{B Y}^{\alpha}$ and $e_{i}^{\alpha}$ are unchanged.

3. A NEW TREATMENT OF THE CONCEPT TIME.

In what follows we will consider real flelds and real coordinates as we have not found anything to be gained by the Minkowski hypothesis.

Essential to our view is the notion that the field equations admit arbitrary data at a point rather than on a hypersurface. Thus, in integrating field equations from the arigin point to some particular point we cannot expect that the integration should 
be independent of path. The integration is only independent of path provided a set of nonlinear algebraic equations are satisfied by the origin point data (Muraskin [1]). The integrablity equations amount to 96 or 384 conditions, depending on whether $R_{j k 1}^{i}=0$ or $R_{j k}^{i} \neq 0$. The integrabillty relations are severely restrictive on the theory and thus can be thought of as unnatural.

In dealing with the no integrability system we have exploited two approaches:

1. An integration path is specifted at the outset.

2. A sumation over path technique is used to integrate the equations.

The sumation over path technique was developed originally in Muraskin [5]. It was also obtained from fundamental considerations in Muraskin [2] and Muraskin [7].

We have enough freedon in the two approaches to introduce a new concept of time which distinguishes time from space in a different manner than the Minkowski hypothesis. In integrating the field frua the origin to another point at $t=0$ we use the sumation over path approach. The results at any space point then evolve in time by integrating along the four dimension. Thus, an integration path is being specified here (approach 1 of above).

This procedure enables us to evaluate the field at any space-time point in a well defined way, and also enables us to conform to the intuitive notion of "flow" to be associated with $t$ lme. At any point at $t=0$ the only way an integration can be performed is in time. This is the way we can express the notion that "time does not stand still." Thus, in order to introduce the flow concept intuitively associated with time it is not necessary to have hyperbolic equations with data prescribed on a hypersurface (in hyperbolic equations the arbitrary data evolves by means of the field equations to succeeding hypersurfaces).

4. A STUDY OF PARTICLE TRAJECTORIES USING THE NEW APPROACH TO TIME.

In Muraskin [4] we studied the data (2.2) when use is made of the superposition principle:

$\Gamma(Q)=-\frac{1}{\text { no. of paths }}$ paths (contributions from each path).

We previously studied the superposition principle in the simplest approxination for which the sumation over contributions does not collapse into a single term. We have called this approximation the naive approxination. Here zig-zag paths are omitted. Although this approximation is not in general reliable, it does give us an opportunity to investigate how a superposition of terms can affect the notion of lattice particles.

When we consider only one path (we use the path prescribed in Section 1) we find that the lattice particles all undergo what appears to be simple harmonic motion. The lattice particles can then be followed for as long a time duration as one pleases, so $\mathrm{far}$ as the numerical work indicates. Such a system we have called a $\mathrm{C}$ systen in Section 1 .

If we use the naive approximation the results are consistent with lattice particles which cannot be followed in time. We then have a $Q$ system. However, in 
Muraskin [4], time is treated in a similar way as space in all respects, and so the flow concept is lost.

The question we address ourselves to is how does the solution behave when we adopt the data (2.2), in the naive approxitnation to (4.1), when $t i d e$ is introduced in the manner set down in section 3.

We cocus attention on a 3 dimensional miniıum located at

$$
x=6 \times 1.2 \quad y=(5 \text { or } 6) \times 1.2 \quad z=(0 \text { or }-1) \times .6 .
$$

The location of the minunum is followed in time. The results are given in Table I. Values of $x$ and $y$ are given in nultiples of 1.2, while those of $z$ and $t$ are given in terins of . 6 .

Table I

\section{Location of Mininum}

$\begin{array}{rrrl}\underline{t} & \underline{z} & \underline{x} & y \\ -2 & -2 & 8 & 3 \\ -1 & -2 & 8 & 4 \\ 0 & 0,-1 & 6 & 5,6 \\ 1 & 0 & 4 & 7 \\ 2 & -1 & 4 & 8 \\ 3 & -2 & 3 & 8 \\ 4 & -2 & 2 & 8 \\ 5 & 3 & -3 & 11 \\ 6 & 2 & -3 & 10,11 \\ 7 & 1 & -4 & 11 \\ 8 & -1 & -4 & 10\end{array}$

\section{Magnitude of Mininum}

$$
\begin{aligned}
& -1.52 \\
& -1.30 \\
& -1.11 \\
& -1.28 \\
& -1.62 \\
& -1.94 \\
& -2.10 \\
& -2.12 \\
& -2.15 \\
& -1.86 \\
& -1.46
\end{aligned}
$$

There is an abrupt change in the location of the particle between $t=4$ and $t=5$. In Table II we subdivide the time interval between $t=4$ and $t=5$ and attempt to follow the particle motion (remember $t=4$ is in units of .6 so its actual value here is 2.4). In Table II, $t$ is the actual value of this quantity while the other quantities are represented in the same manner as in Table $I$.

Table II

$$
t=2.56
$$

Location of Minimun

Magnitude of Minimun

$\begin{array}{rcc}\underline{z} & \underline{x} & \underline{y} \\ 5 & -3,-4 & 11,12 \\ 4 & -3 & 11 \\ 3 & -2 & 11 \\ 2 & -1 & 10 \\ 1 & 0 & 9 \\ 0 & 0 & 9 \\ -1 & 1 & 8,9 \\ -2 & 2 & 8 \\ -3 & 2 & 8\end{array}$

$$
-1.83
$$$$
-1.98
$$$$
-2.01
$$$$
-2.02
$$$$
-2.02
$$$$
-2.06
$$$$
-2.08
$$$$
-2.09
$$$$
-2.04
$$

$t=2.64$ 


\begin{tabular}{|c|c|c|c|}
\hline \multicolumn{3}{|c|}{ Locat isn of Minimun } & \multirow[t]{2}{*}{ Magnitude of $\mathrm{Yi}_{\mathrm{j}}$} \\
\hline$\underline{z}$ & $\underline{x}$ & $\mathrm{y}$ & \\
\hline 4 & -3 & 11 & -2.1 \\
\hline 3 & $-2,-3$ & 11 & -2. \\
\hline 2 & -2 & 10) & -2 \\
\hline 1 & -1 & 9,10 & -2 \\
\hline 0 & 0 & 9 & -2. \\
\hline-1 & 1 & 8,9 & -2 \\
\hline-2 & 2 & 8 & $-2 . c$ \\
\hline-3 & 2 & 8 & -2 \\
\hline
\end{tabular}

$t=2.38$

\begin{tabular}{|c|c|c|c|}
\hline$\underline{2}$ & $\underline{x}$ & $\underline{y}$ & \\
\hline 4 & -3 & 11 & -2.06 \\
\hline 3 & -3 & 10 & -2.10 \\
\hline 2 & -2 & 10 & -2.14 \\
\hline 1 & -1 & 9 & -2.09 \\
\hline 0 & 0 & 9 & -2.08 \\
\hline-1 & 1 & 9 & -2.06 \\
\hline-2 & 1 & 8 & -2.05 \\
\hline-3 & 2 & 8 & -2.00 \\
\hline
\end{tabular}

An abrupt change in path can be a sign that the trajectory is not continuous. Thus, we have subdivided the time period between $t=4 \times .6$ and $t=5 \times .6$. With a .3 grid we see the following picture. At $t=2.56$ there is a roinimun at $z=-2$. At $t=2.64$ we find two minima. At $t=2.88$ the ininirum at $z=-2$ no longer appears. Thus, it appears that the original minimun disappears and another reappears.

In view of possible nunerical errors, there is another way to interpret the abrupt change of the motion of the particle. It may be that we have a single particle having a discontinuous velocity.

The results, thus, may be interpreted in terins of noncontinuous notion. A further study was made at $t=8$. At $t=9$ no minimum $c$ an be found in the region of the ninimun located at $8 \times .6=4.8$. So again we subdivided the time interval. At $t=4.96$ the mininum is at $z=-1, x=-4, y=10$, with nagnitude -1.30 . At $t=5.2$ the minimum is at $z=-1, x=-5, y=9$, with nagnitude -1.26 . However, at $t=5.28$ the rinisura cannot be found in the region. It gets "swamped" by another mininum located at $z=-1, x=-9, y=1,2$. This latter minimun was present at $t=4.8$ as wel1, although its magnitude is less here.

Thest numerical results are consistent with particles having non well-defined trajectories in time, that is a $Q$ system.

If the studies were made, not with the new concept of time, but time treated the same as space, we also get a $Q$ theory. However, then we do not have the flow concept 
associated with time. We see that the $Q$ notion of the theory is not dependent on whether we treat tine the same as space or not.

If we alter $x, y, z$, so they are treated like the new time (for example, the use of path A. in Muraskin [4]) we find that the theory is a C theury.

5. SUMMARY.

The lattice solution of an infinite number of harnonic oscillators can be obtained in the Aesthetic Field Theory. This system of infinite oscillators can be studied to see how altering fundamental concepts can affect the notion of lattice particles.

The Minowski hypothesis treats time differently froin space but did not lead to new kinds of solutions to $(2.1)$. We then looked to another way to treat time different frow space.

We found that the aesthetic approach offered us the possibilty of introducing time in a novel way. We were able to introduce time so it obeys the following properties: the field equations treat all coordinates in a similar way; the flow concept is associated with time but not space; data is not arbitrary on a hypersurface but at a single point.

This concept of time is used in conjunction with the non-trivial superposition principle (4.1). We find, as a solution, a multiparticle systen which is consistent with particles not having well defined trajectorles. We have called this a $Q$ system.

Thus, we have a classical field theory that can be classified as a $Q$ system. The hypothesis, unproved, is that quantun mechanics is a $Q$ system. Further studies of $Q$ systems would thus be of interest.

\section{REFERENCES}

1. MURASKIN, M., Particle Behavior in Aesthetic Field Theory, International Journal of Theoretical Physics 13(1975), 303-316.

2. MURASKIN, M., Non Integrable Aesthetic Fleld Theury, Mathematical and Computer Mode11ing 10(1988), 571-581.

3. MURASKIN, M., Aesthetic Field Theory, A Lattice of Particles, Hardonic Journal $\underline{7}(1984), 296-312$.

4. MURASKIN, M., Trajectories of Lattice Particles, Hadronic Journal Supplement $\underline{2}(1986), 620-639$.

5. MURASKIN, M., Aesthetic Fields Without Integrability, Hardonic Journal 8(1985), 279-285.

6. MURASKIN, M. \& RING, B., A Two Particle Collision in Aesthetic Field Theory, Foundations of Physics 5(1975), 513-523.

7. MURASKIN, M. Sinusoidal Decomposition of the Lattice Solution, Hadronic Journal Supplement 2(1986), 600-619. 


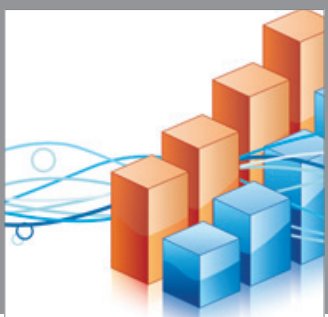

Advances in

Operations Research

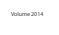

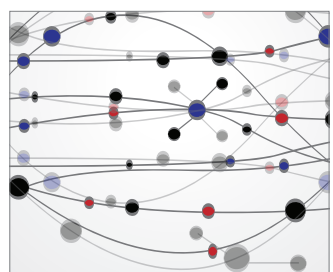

\section{The Scientific} World Journal
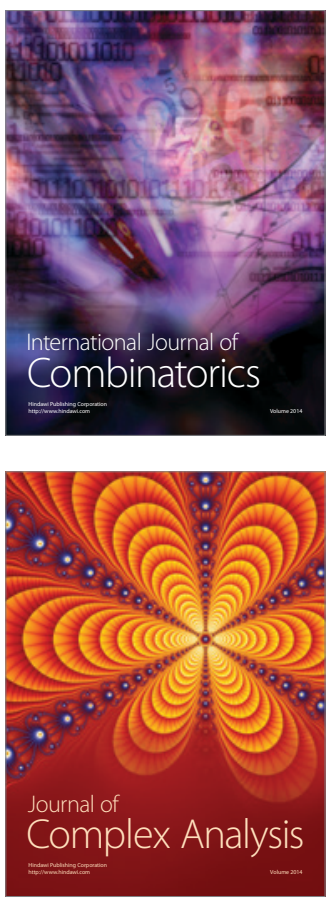

International Journal of

Mathematics and

Mathematical

Sciences
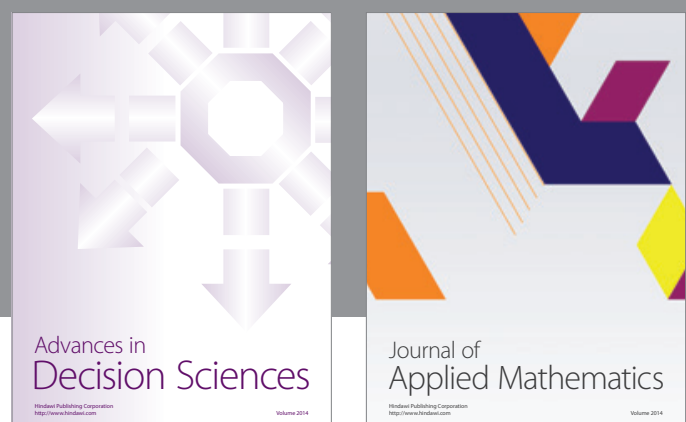

Journal of

Applied Mathematics
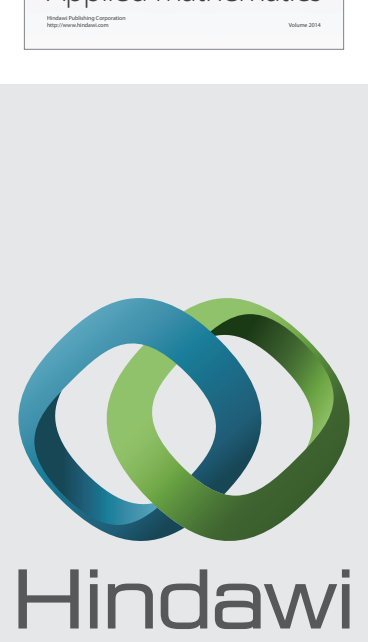

Submit your manuscripts at http://www.hindawi.com
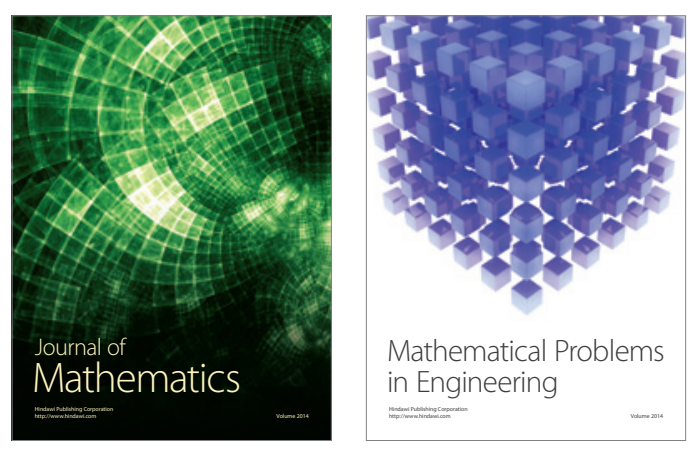

Mathematical Problems in Engineering
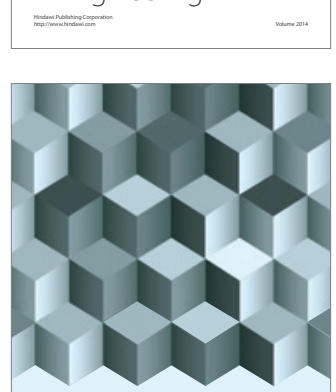

Journal of

Function Spaces
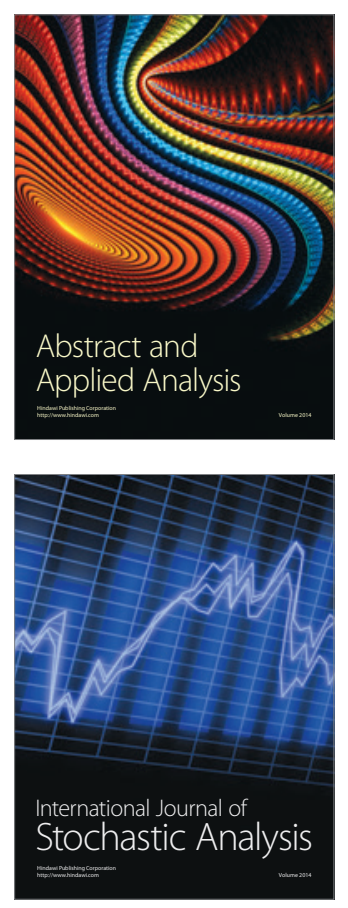

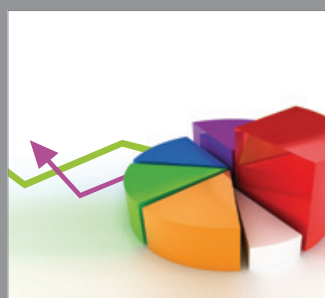

ournal of

Probability and Statistics

Promensencen
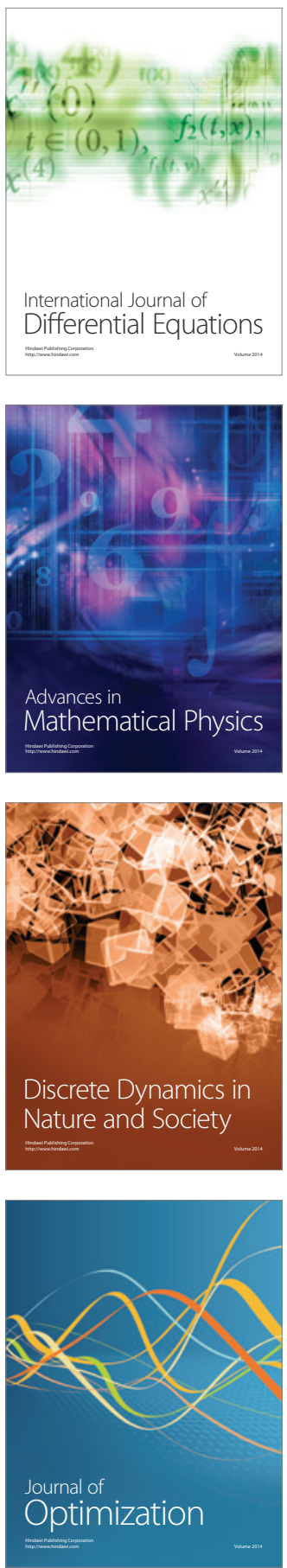